ner politischen Logik ihren Ausdruck findet. Ein solcher ideengeschichtlicher Ansatz scheint jedoch in der Arbeit kaum durch; und die historischen Ausführungen zum Wirtschaften der Parteien, mit denen Schindler ihre Arbeit beginnt, sind für die rechtliche Argumentation eigentlich, wie die Autorin selbst bemerkt, kaum von Belang. Darüber mag man streiten; sie hätten jedoch einen Beitrag leisten können, einen frischen Blick für die Problemlagen auf dem Hintergrund der organisatorischen Vielgestaltigkeit von Parteien zu gewinnen. Die rechtlichen Argumente für eine besondere Obacht gegenüber dem Zusammenhang zwischen wirtschaftlicher und politischer Macht sind bekannt. Dass aber die ökonomische Existenz von Parteien durch Mitgliedsbeiträge, Spenden und staatliche Mittel gesichert sei, verfehlt die spezifischen Schwierigkeiten, denen sich heutzutage Parteien ausgesetzt sehen - zumal ihnen die modernen Formen der Akquise, wie das Beispiel des Sponsoring vor Augen führt, nicht ohne Weiteres zur Verfügung stehen. Nicht nur in dieser Hinsicht hinkt der Vergleich mit dem Idealverein.

Oliver W. Lembcke

\title{
Licht und Schatten der vergleichenden Wahlsystemforschung
}

\author{
Gallagher, Michael und Paul Mitchell (Hrsg.): The Politics of Electoral Systems, Oxford Univer- \\ sity Press, Oxford 2008, 688 Seiten (Paperback), € 34,99 (£ 27,50).
}

Zwei ausgewiesene Wahlexperten, Michael Gallagher und Paul Mitchell, legen ein neues Handbuch zu Wahlsystemen und ihren Auswirkungen vor, das sich allein schon durch seinen Umfang (688 Seiten) und die Zahl seiner Beiträger (31) hervorhebt. ${ }^{1}$ Im Mittelpunkt des Sammelbandes stehen 22 Länderstudien. Sie werden umrahmt von thematisch übergreifenden Beiträgen, einer Zusammenfassung der Ergebnisse und einem umfangreichen dokumentarischen Anhang. Arend Lijphart hat ein sehr wertschätzendes Vorwort geschrieben.

Beide Herausgeber führen zunächst in die technischen Dimensionen der Wahlsysteme und in die Struktur des Handbuches ein. Es folgen zwei Beiträge zu übergreifenden Fragestellungen. Der erste stammt aus der Feder von Matthew S. Shugart und hat die vergleichende Wahlsystemforschung zum Gegenstand, der er einen Prozess der Reife bescheinigt und einige neue Herausforderungen mit auf den Weg gibt. Der zweite ist von Richard S. Katz verfasst und widmet sich der (bereits im Titel provokant formulierten) Frage, warum es so viele (oder so wenige) Wahlreformen gibt. Er klärt darüber auf, dass zwar etliche kleinere Änderungen vorgenommen werden, aber wenig grundsätzliche, und dass überhaupt die Wahrscheinlichkeit von Wahlreformen eher gering sei.

Die Länderbeiträge sind nach Wahlsystemen geordnet, wobei als zentrale Unterscheidungsmerkmale der Einerwahlkreis und die Listenwahl dienen. Denkt man sich die Wahlsysteme auf einem bipolaren Kontinuum angesiedelt, so bilden diese beiden Merkmale die Pole für eine Klassifizierung in vier Gruppen. Die erste ist durch den Einerwahlkreis ge-

1 Das Werk ist im Hardcover 2005 bei Oxford University Press erschienen. Der Besprechung liegt die aktualisierte erste Paperback-Ausgabe von 2008 zugrunde. 
kennzeichnet (Australien, Frankreich, Kanada, Indien, Vereinigtes Königreich, Vereinigte Staaten). In der zweiten Gruppe treten sowohl der Einerwahlkreis als auch die Liste auf (Deutschland, Italien, Japan, Neuseeland, Russland, Ungarn). Die dritte hat die starre Liste zum Merkmal (Israel, Spanien und Südafrika). In der vierten Gruppe schließlich herrscht die lose gebundene Liste vor (Belgien, Chile, Dänemark, Finnland, Niederlande, Österreich), in die das System übertragbarer Einzelstimmgebung (Irland) eingebunden wird.

Die Länderanalysen folgen einer vorgegebenen Inhaltsstruktur. Zunächst wird der historisch-politische Hintergrund eines Landes dargestellt, dann die Genesis des gegenwärtig gültigen Wahlsystems behandelt und anschließend dessen Funktionsweise erläutert. Im zentralen Teil der Länderbeiträge wird systematisch in Einzelabschnitten nach den Auswirkungen der Wahlsysteme auf das Parteiensystem, die Parteien, das Parlament und die Regierung(sbildung) gefragt. Schließlich werden unter der Kapitelüberschrift „The Politics of Electoral Reform" Reformperspektiven erörtert.

Das Handbuch stellt ohne Zweifel ein wichtiges Kompendium der Wahlsystemforschung dar. Es überzeugt durch den „comprehensive approach“, der sich darin äußert, dass die technischen Aspekte von Wahlsystemen und deren Auswirkungen im jeweils nationalen Kontext untersucht werden. Der Band kann weiterhin durch die Fülle systematisch aufbereiteter Informationen gefallen, was insbesondere in der reichen Verwendung von Tabellen und Abbildungen sichtbar wird. Hervorzuheben ist auch, dass die Entstehung der Wahlsysteme gebührend berücksichtigt und damit einem komplexeren Verständnis des Zusammenhangs von Wahl- und Parteiensystemen gedient wird. Darüber hinaus nehmen viele der erzielten Ergebnisse, sowohl in den Länderstudien als auch in den übergreifenden Beiträgen, für ihn ein. Dies gilt vor allem für die Bedeutung, die Kontextvariablen für die Auswirkungen von Wahlsystemen zugesprochen wird.

Allerdings sind viele Ergebnisse vielfach nicht so neu, wie sie sich geben, sondern eher eine Bestätigung von Erkenntnissen, zu denen beispielsweise die deutsche Wahlsystemforschung bereits vor Jahren gelangt ist. Deren Untersuchungen werden aber bis auf wenige Ausnahmen sichtbar nicht zur Kenntnis genommen. Internationale Wahlsystemforschung bestätigt sich in diesem Band einmal mehr als englischsprachige Forschung. Das hat seinen Preis: Nicht nur deutsche Studien, sondern auch französisch und spanisch verfasste Untersuchungen bleiben fast gänzlich unberücksichtigt, zudem erfolgen eher Erkenntnis verschüttende Anpassungen an die im englischen Sprachraum gängige Terminologie. So kommt der Begriff der personalisierten Verhältniswahl im ganzen Buch nicht vor, auch nicht im Deutschland gewidmeten Beitrag, obwohl dem deutschen Wahlsystem Modellcharakter für viele Reformprozesse zugeschrieben wird. Folglich wird auch das deutsche Wahlsystem nicht als Verhältniswahl geführt, sondern als Mischsystem, als „archtype of a mixed-member proportional system“ (S. 209). Seiner richtigen Typisierung als ,a system of proportional representation (...) with a personalized element" (S. 212) widerspricht Thomas Saalfeld in seinem Beitrag leider kontinuierlich selbst, indem er sich der britischen Sicht auf das deutsche Wahlsystem beugt. Das geht sogar so weit, dass er als Verrechnungsmethode nicht das Verfahren Hare/Niemeyer nennt, sondern LR-Hare (S. 214), also das Hare'sche Wahlzahlverfahren mit größtem Überrest, das die Mandatsverteilung anders berechnet, aber im Ergebnis dem HarelNiemeyer-Verfahren gleicht.

Die im Handbuch getroffene Auswahl der Fälle ist natürlich für nichts repräsentativ. In einem Sammelband kann auch kein komparatives Forschungsdesign erwartet werden. Es fällt allerdings eine Konzentration auf die westlichen Industriestaaten auf. Ausnahmen bil- 
den nur Chile, Indien, Russland, Ungarn und Südafrika. Alle westlichen Länder, in denen nach einem klassischen Mehrheitswahlsystem (relativ oder absolut) gewählt wird, sind vertreten, verständlicherweise nur eine Auswahl von Verhältniswahlsystemen. Starke Berücksichtigung finden Staaten, in denen es in den letzten Jahren zu einem grundlegenden Wahlsystemwechsel gekommen ist: Italien, Japan, Neuseeland - zweifelsohne sinnvoll, denn solche Wechsel bilden Gelegenheiten, in denen die Auswirkungen unterschiedlicher Wahlsysteme unter sonst annähernd gleichen Bedingungen gut zu studieren sind. Tendenziell unterrepräsentiert sind Länder, die im Transitionsprozess zu ihrem Wahlsystem gefunden haben, zumal da deren Genese neuerdings große Beachtung geschenkt wird.

Natürlich sind die Länderexpertisen unterschiedlichen Niveaus, vor allem mit Blick auf die Wahlsystemfrage. Nicht jeder Länderspezialist bringt in dieser speziellen Hinsicht auch komparative Erfahrung mit. Mancher tut sich bereits in der Darstellung des Wahlsystems schwer, etwa im Falle Russland. Hier dehnt Steven White den Begriff Wahlsystem zunächst aus, dann vermengt er Informationen zum Präsidentschafts- und Parlamentswahlsystem, und schließlich mündet die Darstellung in den Titel „Russia: The Authoritarian Adaption of an Electoral System“, das heißt, in einer Aufgabe der Unterscheidung zwischen System und Umwelt. Die Titel der Länderbeiträge verraten auch unterschiedliche Akzente, legen kein einheitliches Untersuchungsziel nahe.

Klassifizierung ist für die Wahlsystemforschung von allergrößter Bedeutung. Nur auf diese Weise lassen sich verallgemeinerbare Aussagen über die Auswirkungen von Wahlsystemen treffen. Im vorliegenden Handbuch wird der problematische Weg eingeschlagen, einige technische Elemente als Unterscheidungskriterien zu verwenden, was nicht neu ist, aber in der Forschung bereits häufiger verworfen wurde. Damit wird die Orientierung an den Repräsentationsprinzipien der Mehrheitswahl (die Bildung von Mehrheiten im Parlament zu fördern) und der Verhältniswahl (eine möglichst proportionale Repräsentation zustandezubringen) ebenso wie klassische Unterscheidungen zwischen verschiedenen Typen von Mehrheitswahl und Verhältniswahl aufgegeben. In der vorliegenden Typologie fallen so relative und absolute Mehrheitswahl in eine Klasse, obwohl ihnen bekanntlich sehr unterschiedliche Wirkungen beizumessen sind. Das Kriterium der Wahlkreisgröße, die als entscheidender Faktor in der Wahlsystemforschung erkannt worden ist, wird aber anschließend fallengelassen. Als Unterscheidungskriterien werden die Formen der Listen in den Vordergrund gerückt, die für die Auswirkungen der Wahlsysteme auf die parteipolitische Komposition der Parlamente nachrangig sind. In ein und derselben Kategorie der „mixed systems“ werden Wahlsysteme zusammengefasst, die in ihren Effekten entweder mehr der Mehrheitswahl oder mehr der Verhältniswahl zuneigen. Die gleiche Beobachtung trifft für die Listensysteme zu - besonders sichtbar in den Titeln der Beiträge zu Ländern mit starren Listen: Südafrika als System der „Perfect Proportionality“, Spanien als „Proportional Representation with Majoritarian Outcomes“. Sinnvoller wäre es gewesen, die Wahlsysteme nach den in der Gesamtgestaltung ihrer Einzelelemente ruhenden mutmaßlichen Auswirkungen zu klassifizieren.

Das Zurück zu den Gestaltungselementen als Klassifikationskriterien von Wahlsystemen bestätigt keineswegs das positive Bild, das Matthew S. Shugart von der Entwicklung der internationalen Wahlforschung zeichnet. Gewiss ist unser Wissen über Wahlsysteme erheblich angewachsen - und der vorliegende Band trägt weiter dazu bei. Aber sind wir in den konzeptionellen und theoretischen Fragen wirklich weiter gekommen? „The Politics of Electoral Systems“ verstärkt eher den gegenteiligen Eindruck. Kein Autor wird in diesem 
Band so stark in Anspruch genommen wie Maurice Duverger. Als hätte eine ganze Forschungsrichtung nichts anderes zu tun, als immer wieder die Thesen zu widerlegen, von denen sich Duverger vielfach selbst distanziert hat. Erfreulich ist natürlich, dass etliche frühere Forschungsergebnisse bestätigt werden, unter anderem die These des Rezensenten aus dem Jahre 1984, dass grundlegende Wahlsystemwechsel rar sind. Man sollte sich jedoch davor hüten, den national begrenzten Vergleich, wie Matthew S. Shugart (S. 29), auch als komparativ zu bezeichnen, der im Grunde deshalb vorzuziehen sei, weil in ihm - anders als im Ländervergleich - viele Variablen konstant gehalten werden könnten. Zum einen wird vernachlässigt, worin Kunst und Gewinn komparativer Forschung liegen: die Kunst im qualitativen Forschungsdesign ausgesuchter Fälle, die ein analytisch fruchtbares Maß an Ähnlichkeiten und Unterschieden aufweisen, der Gewinn in der näheren Bestimmung jener gesellschaftlichen und politischen Bedingungen, unter denen Wahlsysteme diese oder jene Auswirkungen zeigen. In einer qualitativ vergleichenden Analyse werden dabei unabdingbar die wechselhaften Konsequenzen der Kontextfaktoren thematisiert, die in einer nationalen Untersuchung leichthin als konstant angenommen werden und dann systematisch außerhalb der Untersuchung bleiben. Zum anderen ist es diese Annahme selbst, dass der nationale Kontext für die Wahlsystemanalyse über längere Zeit konstant bleibe, die zu den üblichen Reduktionismen der Wahlsystemforschung gehört.

Dieter Nohlen

\section{Prominente Studie zu politischen Milieus}

Neugebauer, Gero: Politische Milieus in Deutschland. Die Studie der Friedrich-Ebert-Stiftung, Verlag J.H.W. Dietz Nachfolger, Bonn 2007, 145 Seiten, € 9,90.

Sozialwissenschaftlichen Studien wird in der Regel nur begrenzte Aufmerksamkeit geschenkt. Einige Arbeiten erfahren eine gewisse fachinterne Resonanz, aber nur ganz wenige werden von einer breiteren Öffentlichkeit wahrgenommen oder gar Gegenstand politischer Diskussionen. Dass aber eine Untersuchung bereits vor ihrem eigentlichen Erscheinen eine unmittelbare Reaktion des Vizekanzlers hervorruft, ist wohl ein Unikum und besonderen Entstehungszusammenhängen geschuldet. Im Herbst 2006 fügte sich der Befund einer von der Friedrich-Ebert-Stiftung in Auftrag gegebenen Studie, wonach acht Prozent der Bundesbürger dem „abgehängten Prekariat" ${ }^{\text {" }}$ angehören, genau in die vom damaligen SPDVorsitzenden Kurt Beck initiierte so genannte Unterschichten-Diskussion. Die Untersuchung wurde als Beleg für die Verschärfung sozialer Probleme infolge der von der SPD zu verantwortenden „Agenda 2010“-Politik gewertet. Der damalige Arbeits- und Sozialminister Franz Müntefering sah sich daraufhin zu der Aussage veranlasst, es gebe keine Schichten in Deutschland, sondern lediglich Menschen, die schwächer seien und es schwerer hätten.

Nachdem die öffentliche Aufmerksamkeit sich wieder anderen Themen zugewendet hat, lohnt es sich, jenseits tagespolitischer Aufgeregtheiten einen zweiten Blick auf die Studie

1 So die Begriffsbildung von Rita Müller-Hilmer vom Institut TNS Infratest Sozialforschung, das wesentlich an der Studie beteiligt war. 